\title{
Characterization and Cell Culture of a Grafted Chitosan Scaffold for Tissue Engineering
}

\author{
Wen-Chuan Hsieh, Jiun-Jia Liau, and Yi-Jhong Li \\ Department of Biological Science and Technology, I-Shou University, Kaohsiung 82445, Taiwan \\ Correspondence should be addressed to Wen-Chuan Hsieh; wenchuan@isu.edu.tw
}

Received 21 October 2014; Accepted 24 December 2014

Academic Editor: Huaping Tan

Copyright ( 2015 Wen-Chuan Hsieh et al. This is an open access article distributed under the Creative Commons Attribution License, which permits unrestricted use, distribution, and reproduction in any medium, provided the original work is properly cited.

Poly(vinyl alcohol) (PVA) was grafted to chitosan to form a porous scaffold. The PVA-g-chitosan 3D scaffold was then observed by Fourier transform infrared spectroscopy (FT-IR). The water absorbency of PVA-g-chitosan was increased $370 \%$ by grafting. Scanning electron microscope (SEM) observations of the material revealed that the 3D scaffold is highly porous when formed using a homogenizer at $300 \mathrm{rpm}$. Compression testing demonstrated that as the amount of chitosan increases, the strength of the 3D scaffold strength reached showed that, by increasing the amount of chitosan, the strength of the 3D scaffold could be increased to $16 \times 10^{-1} \mathrm{MPa}$. Over 35 days of enzymatic degradation, the 3D scaffold was degraded by various enzymes at rates of up to $10 \%$. In vitro tests showed good cell proliferation and growth in the $3 \mathrm{D}$ scaffold.

\section{Introduction}

Chitin can be extracted and purified from the shells of shrimp, crab, and other crustaceans and from the cell walls of some fungi. Chitin is a deposit of natural cationic polysaccharides, and its abundance is second only to that of cellulose. Chemical modifications of the amino and hydroxyl groups in its molecular structure can easily produce other derivatives. Chitosan can be formed into gels, orbs, fibers, and membranes for various uses. Moreover, because chitosan is easily obtained and conforms to the requirements of many tissue engineering applications, it can be implanted into the human body without harm. Accordingly, the material is widely used in tissue engineering [1].

However, since chitosan alone is insufficiently strong to support cell growth, a method of enhancing its mechanical strength is needed. Most of the numerous studies of direct applications of chitosan in 3D scaffolds have focused on blending 3D composite scaffolds with other materials for permanent implantation in the human body $[2,3]$. Chitosan has been chemically modified for use in
3D scaffolds such as chitosan hydrogels, chitosan sponges, and 2D scaffolds such as chitosan films, nanofiber membranes, by way of sulfonation, phosphorylation, quaternization, and cross-linking methods [4-11]. However, this study is the first to apply a grafting method in a 3D scaffold.

Another biodegradable polymer used in tissue engineering is PVA. Production methods include chemical synthesis $[1,2]$, melt-molding particulate-leaching [3], freeze-drying [12-15], and electrospinning to form nanofibrous scaffolds $[16,17]$. Therefore, the goal of this study was to exploit an existing, inexpensive, and biodegradable material to form a $3 \mathrm{D}$ scaffold for use in regenerative medicine. Because PVA is easy to obtain and highly biodegradable and biocompatible, PVA and chitosan are considered herein. Chitosan and PVA both are biodegradable, biocompatible, and easy to obtain. No complex or expensive equipment is needed. In fact, a chemical grafting method can be used to form the 3D scaffold and to improve its mechanical properties and formation. This study elucidated the structural changes in this material, including its swelling, formation, mechanical 
properties, biodegradability, and porosity. Finally, NIH3T3 fibroblasts cells were transplanted and cultivated to form a 3D scaffold. The potential use of the material for tissue engineering was then evaluated.

\section{Materials and Methods}

\subsection{Materials}

2.1.1. Materials. Poly(vinyl alcohol) (PVA) was purchased from Sigma-Aldrich, average molecular weight (Mw) 85,000-124,000 g/mol, 87-89\%, and was hydrolyzed and used without further treatment or purification. Chitosan $[\operatorname{poly}(\beta-$ (1-4)-2-amino-2-deoxy-D-glucopyranose)] (75\% degree of deacetylation) and formaldehyde were obtained from the Sigma-Aldrich. Sulfuric acid $\left(\mathrm{H}_{2} \mathrm{SO}_{4}\right)$ was purchased from The First Chemical Company. All materials and chemicals were used as acquired with no further processing.

2.1.2. Preparation of $3 D$ Scaffold. The chitosan powder $(0.25$, 0.5 , and $0.75 \mathrm{~g}$ ) (name: $\mathrm{CH} 25, \mathrm{CH} 50$, and $\mathrm{CH} 75$ ) was separately dissolved in $1 \%$ acetic acid $(20 \mathrm{~mL})$ at room temperature. The PVA ( $4 \mathrm{~g}$ ) was dissolved completely in RO water $(20 \mathrm{~mL})$ by heating. The chitosan solution was added to the PVA solution and mixed uniformly with a homogenizer at $300 \mathrm{rpm}$ and at $90^{\circ} \mathrm{C}$ for 30 minutes until the mixture was in a colloidal state. After adding $\mathrm{H}_{2} \mathrm{SO}_{4}(10 \mathrm{~mL})$ and formaldehyde $(5 \mathrm{~mL})$ and stirring, the sample was cooled to room temperature. Finally, the sample was poured into a tube and heated in an oven at $60^{\circ} \mathrm{C}$ for 50 minutes. The $3 \mathrm{D}$ scaffold was washed with water to remove residual agents.

\subsection{Methods}

2.2.1. SEM Analysis. A field emission scanning electron microscope (FE-SEM Hitachi-4700, Japan) was used for morphological study of the 3D scaffold. The blank sample was completely dried and then fastened to a copper base with conductive tape. After plating with platinum in a vacuum deposition machine, the sample was placed on the sample base of the FE-SEM for observation under an accelerating voltage of $10 \mathrm{kV}$ at a working distance (WD) of $15 \mathrm{~mm}$. All enzyme-degraded samples were photographed after the treatment processes. The 3D scaffold sample with the cultured cells was dehydrated in a paraformaldehyde solution for 12 hours. The dehydrated sample was then freeze-dried, and the surface of the sample was analyzed by energy dispersive spectroscopy (EDS) (HORIBA, Japan).

2.2.2. FT-IR Analysis. The specific chemical groups of the graft scaffolds were analyzed with FT-IR (Perkin Elmer FTIR Spectrum One, USA). The PVA, chitosan, and grafted PVA-g-chitosan 3D scaffold were dehydrated in an oven and then analyzed by FT-IR (powder was using $\mathrm{KBr}$ and solid was using ATR method). The $400 \mathrm{~cm}^{-1}-4000 \mathrm{~cm}^{-1}$ spectrum was scanned and recorded to identify the effects of the above process on functional groups in the PVA-g-chitosan 3D scaffolds.
2.2.3. Compression Strength Test. The mechanical properties of PVA-g-chitosan 3D scaffolds on the wetting state were examined by measuring compression strength (Shimadzu EZ tensile, Japan) under JIS K7181 (ASTM 695, ISO 604). During the compressive strength tests, stress and strain responses were also monitored.

Compression strength was tested in $\psi 14 \mathrm{~mm} \times H 10 \mathrm{~mm}$ samples under a load of $500 \mathrm{~N}$ and at a rate of $5 \mathrm{~mm} / \mathrm{min}$. Variations in the strain and stress of the scaffold were investigated as the maximum breaking strength was approached. Five samples were tested to obtain an average value and standard deviation.

2.2.4. Porosity. The liquid displacement method was used to measure the porosity of the PVA-g-chitosan 3D scaffold [18]. The volume of the scaffold before it was submerged in water for 30 minutes was designated $V 1$. The total volume of the liquid and the liquid impregnated scaffold was designated $V 2$. After removal of the liquid impregnated scaffold, the remaining liquid volume was designated $V 3$, and the porosity was calculated as follows:

$$
\operatorname{Porosity}(\%)=\left[\frac{(V 1-V 3)}{(V 2-V 3)}\right] \times 100 .
$$

2.2.5. Swelling Ratio of PVA-g-Chitosan Scaffold. The grafted PVA-g-chitosan $3 \mathrm{D}$ scaffold was oven dried at $50^{\circ} \mathrm{C}$. After drying in an oven at $50^{\circ} \mathrm{C}$, the PVA-g-chitosan 3D scaffold was immersed in distilled water at room temperature for 30 minutes. Excess water was wiped from the swollen saturated PVA-g-chitosan 3D scaffold, and the swelling ratio was calculated using the following formula:

$$
\text { Swelling ratio }(\%)=\left[\frac{\left(G_{s}-G_{i}\right)}{G_{i}}\right] \times 100,
$$

where $G_{i}$ represents the initial weight of the freeze-dried PVA-g-chitosan 3D scaffold and $G_{s}$ is the weight of the PVAg-chitosan $3 \mathrm{D}$ scaffold in the swollen state.

2.2.6. Biodegradability. The next test measured the biodegradability of the PVA-g-chitosan 3D scaffold over time. Two enzymes were used to simulate the environment in the human body: lipase $(20 \mu \mathrm{g} / \mathrm{mL})$ and lysozyme $(20 \mu \mathrm{g} / \mathrm{mL})$. Each processed $3 \mathrm{D}$ scaffold (approximate thickness, $5 \mathrm{~mm}$ ) was placed in a $15 \mathrm{~mL}$ centrifuge tube with $1 \mathrm{~mL}$ of PBS buffer solution that could be used with the various enzymes. Varying concentrations of the enzymes were then added. For the in vitro test, the centrifuge tube was placed in a $37^{\circ} \mathrm{C} 100 \mathrm{rpm}$ vibrating thermostatic bath for 28 days. The $3 \mathrm{D}$ scaffold was removed every 7 days and dried in a $25^{\circ} \mathrm{C}$ environment. After each drying step, the weight loss was measured using the following formula:

$$
\text { Weight loss }(\%)=\frac{\left(W_{0}-W_{t}\right)}{W_{0}} \times 100 \% \text {, }
$$

where $W_{0}$ and $W_{t}$ are the scaffold weights before and after the degradation, respectively. 
2.2.7. In Vitro Test. The NIH3T3 fibroblasts were cultured in a Petri dish (diameter, $10 \mathrm{~cm}$ ). The culture liquid contained DMEM (Dulbecco's modified Eagle's medium), 10\% fortified bovine calf serum (FBS), and $1 \%$ penicillin streptomycin solution. The $\mathrm{pH}$ value was maintained at 7.4. The incubator settings were $37^{\circ} \mathrm{C}$ with $5 \% \mathrm{CO}_{2}$ and $95 \%$ relative humidity. The cell culture of PVA-g-chitosan scaffold cycles lasted for three days. A growth curve was plotted based on the changes in cell number, which was counted every 24 hours.

The cells that detached well when trypsinized were placed in a $15 \mathrm{~mL}$ centrifuge; the medium was added in an amount equal to that of trypsin. The mixture was centrifuged at $300 \mathrm{rcf}$ and $4^{\circ} \mathrm{C}$ for 5 minutes. After removing the supernatant, $2 \mathrm{~mL}$ of medium was added to form the cell solution. Approximately $\psi 14 \mathrm{~mm} \times H 1 \mathrm{~mm}$ of scaffold was placed in each well of a 24 -well plate, and $500 \lambda$ of cell solution was added to the surface of the 3D scaffold in each well. After 30 -minute incubation with $5 \% \mathrm{CO}_{2}$ at $95 \%$ relative humidity at $37^{\circ} \mathrm{C}$, the scaffold was removed from the incubator and placed into another well with no additional agents; $2 \mathrm{~mL}$ of medium was added to initiate the cell attachment experiment. After 3 days of culturing, the test sample for EDS analysis was prepared by immersing the scaffold containing the cells in $5 \mathrm{~mL} 10 \%$ formaldehyde overnight to fix the cells to the scaffold.

\section{Results and Discussion}

3.1. FT-IR Analysis of PVA-g-Chitosan. The FT-IR was used to confirm the specific chemical groups of grafted PVA-gchitosan 3D scaffold. Figure 1 displays the FT-IR spectra of PVA, chitosan, and the grafted PVA-g-chitosan 3D scaffold. The observed absorption peaks mainly of PVA were 2941, $1000 \mathrm{~cm}^{-1}$ of $\mathrm{CH}_{2} ; 1734 \mathrm{~cm}^{-1}$ of $\mathrm{C}=\mathrm{O}, 3383$; and $1150 \mathrm{~cm}^{-1}$ of $\mathrm{OH}, 1098 \mathrm{~cm}^{-1}$ of $\mathrm{C}-\mathrm{O}$, and $850 \mathrm{~cm}^{-1}$ of $\mathrm{C}-\mathrm{C}[5,11]$. The functional groups of PVA in the FT-IR spectrum of the grafted PVA-g-chitosan 3D scaffold were also observed. The major absorption peaks of chitosan were $1654 \mathrm{~cm}^{-1}$ of $\mathrm{C}=\mathrm{O}$ NHR and $1540 \mathrm{~cm}^{-1}$ of $-\mathrm{NH}_{2}[19,20]$. The FT-IR peak near $3000 \mathrm{~cm}^{-1}$ was weaker in the $\mathrm{CH}_{2}$ group of chitosan and grafted sample compared to the $\mathrm{CH}_{2}$ group of PVA. The absorption peaks of PVA-g-chitosan at $800-1700 \mathrm{~cm}^{-1}$ were characterized for both PVA and chitosan. The grafting agent formaldehyde (aldehyde group) is known to react with PVA and chitosan (hydroxyl groups) in the presence of an acid catalyst and form 1,3-dioxane rings [21-23]. The successful preparation of the PVA-g-chitosan was confirmed by the significant changes in the major FT-IR absorption peaks of the grafted PVA-g-chitosan upon grafting.

3.2. Morphology of 3D Scaffold. Because of its insufficient strength when incubation time is prolonged, chitosan requires a blend/graft material to support the cell culture. Therefore, the PVA was combined with chitosan to improve its physical properties [8]. The morphology of the grafted PVA-g-chitosan 3D scaffold was then observed by SEM. Figure 2 is a SEM image of the PVA-g-chitosan 3D scaffold. The photographs show the distribution of the pores of the

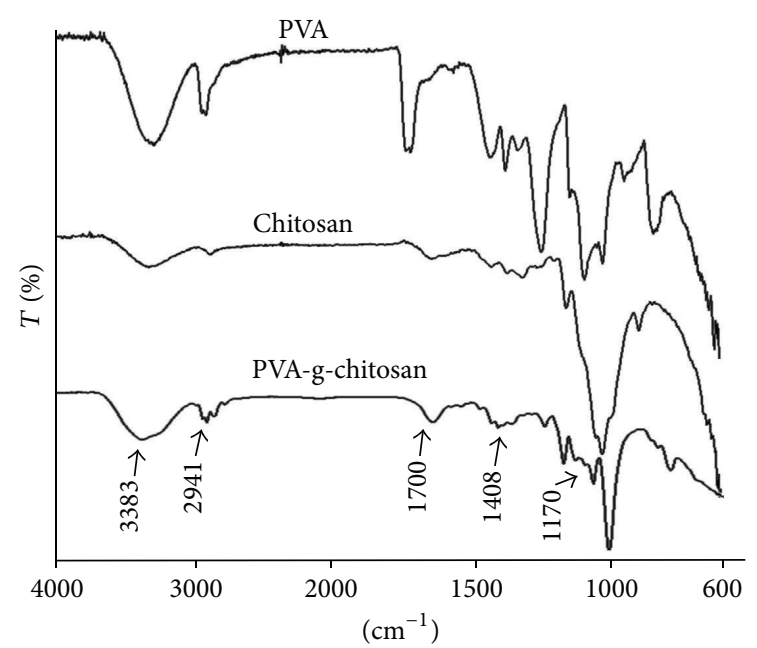

FIGURE 1: FT-IR spectrum of PVA, chitosan, and PVA-g-chitosan 3D scaffold.

grafted PVA-g-chitosan 3D scaffold. The PVA-g-chitosan scaffold in Figure 1 was treated with chemistry foaming agent, which was stirred during the manufacturing process. The resulting 3D scaffolds samples had pore sizes of $\mathrm{CH} 25$ : $452 \mu \mathrm{m}$, CH50: $428 \mu \mathrm{m}$, and CH75: $280 \mu \mathrm{m}$. Apparently, the high concentration of chitosan decreased the pore size in the 3D scaffold.

3.3. Porosity and Swelling Ratio. Figure 3 presents the porosities ( $\square$ ) and swelling ratios ( $\square$ ) of PVA-g-chitosan 3D scaffolds under various concentrations. Porosity is calculated from (1) in Section 2.2.4. Porosity was measured at three different concentrations of the chitosan used to form the 3D scaffold: $85 \%, 75 \%$, and $72 \%$. Figure 3 shows that porosity decreases as the chitosan concentration increases [24]. Notably, regardless of concentration, a high porosity (above $70 \%$ ) could still be maintained in the chitosan scaffolds by controlling the concentration of chitosan and the stirring rate of homogenizer. These materials were then used for cell cultivation to evaluate their physical properties.

The water absorption of the raw materials affected not only the scaffold shape, but also the cell growth. If the cell cultivation processes used in tissue engineering have a long culture time and use highly absorbent materials, the scaffold can become saturated with water and expand, causing deformation and affecting the proliferation and division of cells.

Figure 3 is a diagram of the water absorption in various concentrations of chitosan scaffolds at $300 \mathrm{rpm}$ stirring rates. The ( $\square$ ) represents the swelling ratio. The above histogram is an actual photograph of the PVA-g-chitosan $3 \mathrm{D}$ scaffold during formation. The change ratio of water absorption is the water absorption in relation to the chitosan concentration, which reveals the effects of chitosan concentration on water absorption. The diagram illustrates that, as the concentration of the chitosan solution increases, the change ratio of water absorption decreases. When the ratio of PVA to chitosan was 1:0.25 ( $\mathrm{CH} 25)$, the swelling ratio was $370 \%$. As the chitosan concentration increased ( $\mathrm{CH} 75)$, the absorption of 

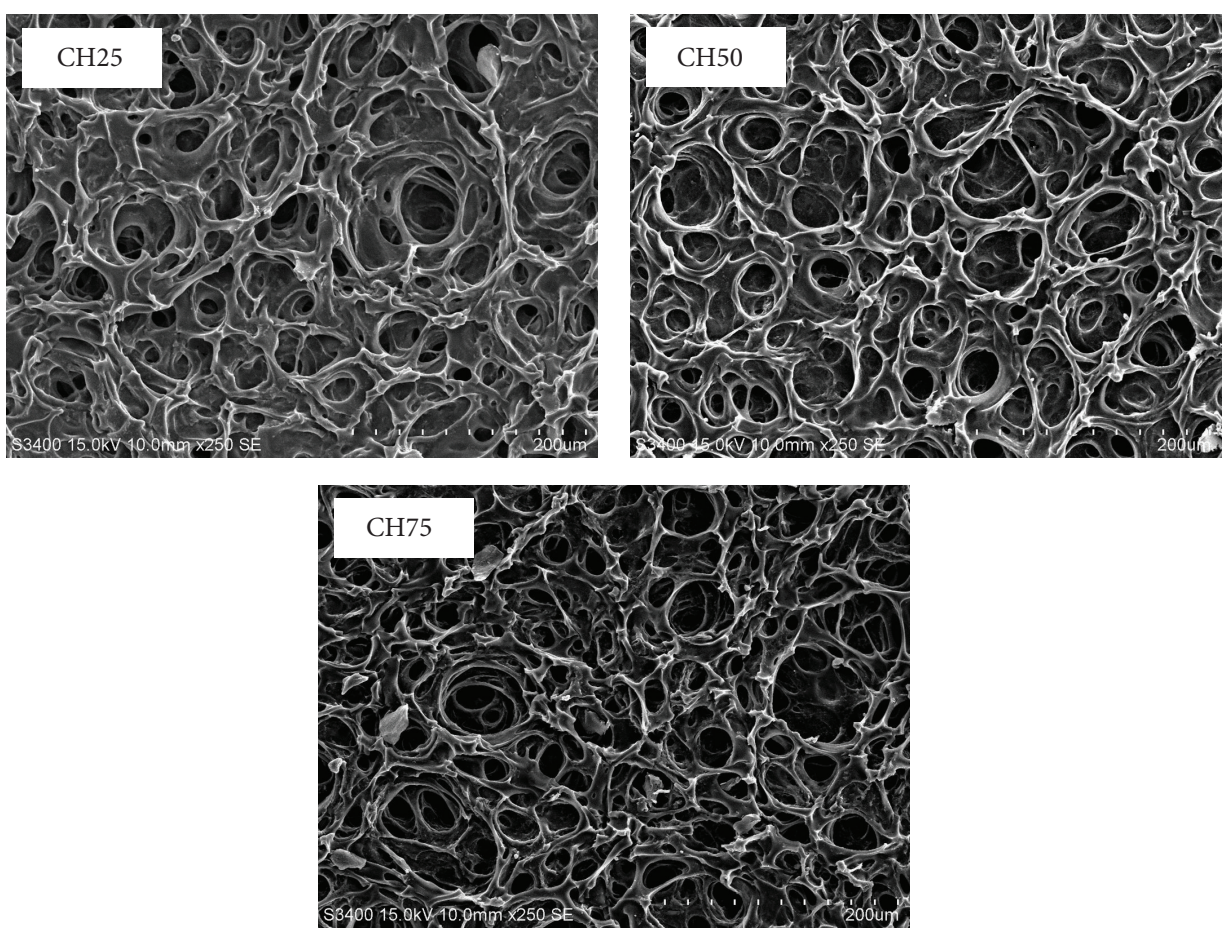

FIGURE 2: SEM image of various grafted PVA-g-chitosan 3D scaffolds. (Chitosan ratio: $\mathrm{CH} 25$ is $0.25 \mathrm{~g}$, $\mathrm{CH} 50$ is $0.50 \mathrm{~g}$, and $\mathrm{CH} 75$ is $0.75 \mathrm{~g}$.)

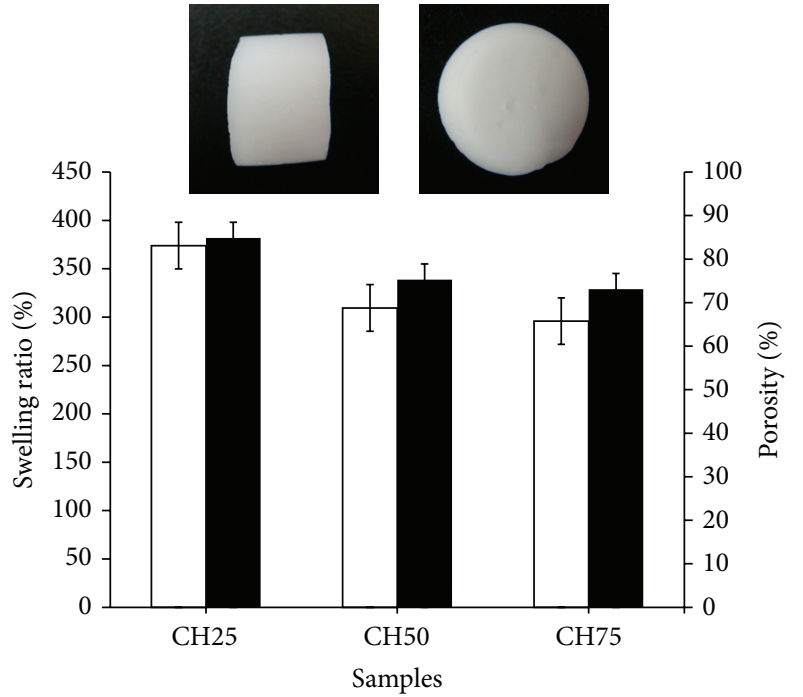

口 Swelling (\%)

- Porosity (\%)

FIGURE 3: Porosity and swelling ratio of various grafted PVA-gchitosan 3D scaffolds. The above histogram is the actual forming figure of the grafted PVA-g-chitosan 3D scaffold.

water considerably decreased to $300 \%$ because, at a high concentration of chitosan, the volume occupied per unit area is high. Therefore, water absorption is much lower than that at low chitosan concentrations.

3.4. Compression Test. During cell cultivation, 3D scaffolds must have sufficient strength to support cell attachment.

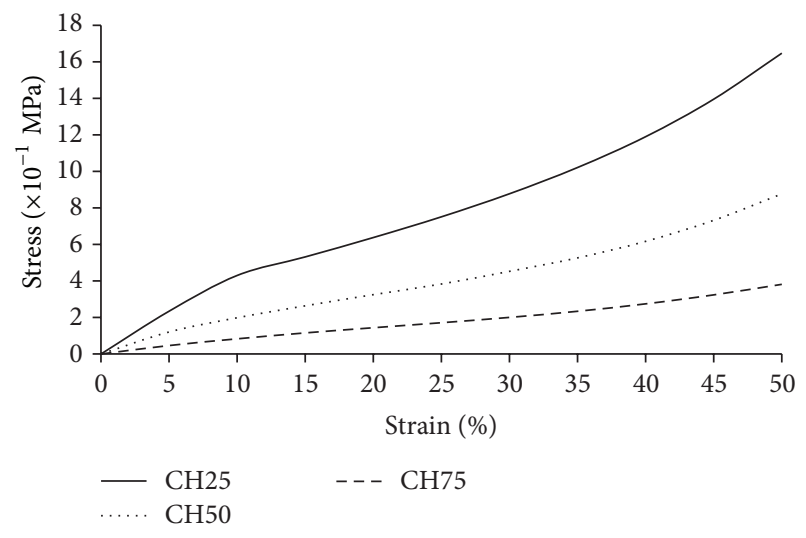

FIGURE 4: The mechanical properties of PVA-g-chitosan 3D scaffolds.

However, a water-swellable 3D scaffold can damage the frame structure of the cell culture when using liquid medium. Therefore, 3D scaffolds must withstand moderate intensity force.

Figure 4 shows the mechanical properties of the 3D scaffold. As the chitosan concentration increased, the mechanical strength of the 3D scaffold decreased. The mechanical strength of 3D scaffolds depends on the chitosan concentration because a 3D scaffold with a high concentration has a hard and brittle structure that can withstand lower mechanical strength. The compression strength of $\mathrm{CH} 25$ PVA-g-chitosan $3 \mathrm{D}$ scaffolds reached $16 \times 10^{-1} \mathrm{MPa}$. In an earlier study, the authors found that the compression strength of pure chitosan 3D scaffold [25] was much lower than that of grafted 3D scaffold. 

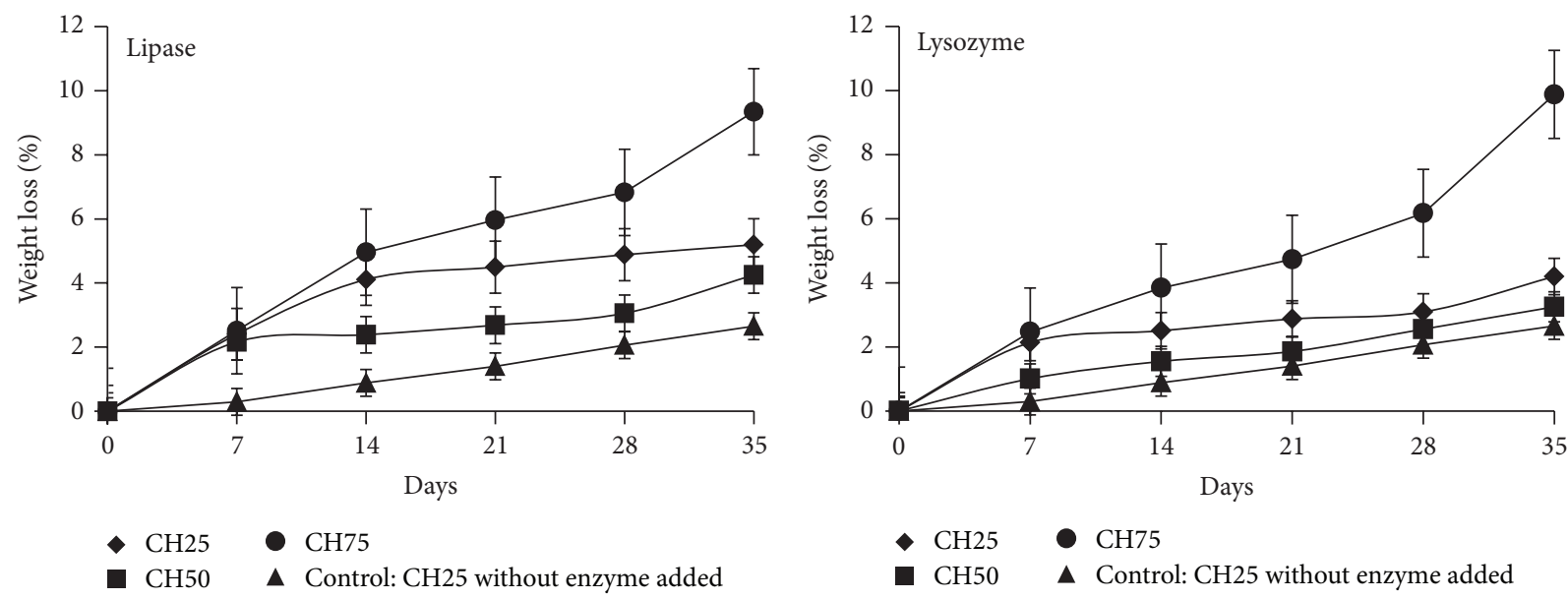

FIGURE 5: Weight loss of various grafted PVA-g-chitosan 3D scaffolds in the various enzymatic degradation at 35 days.

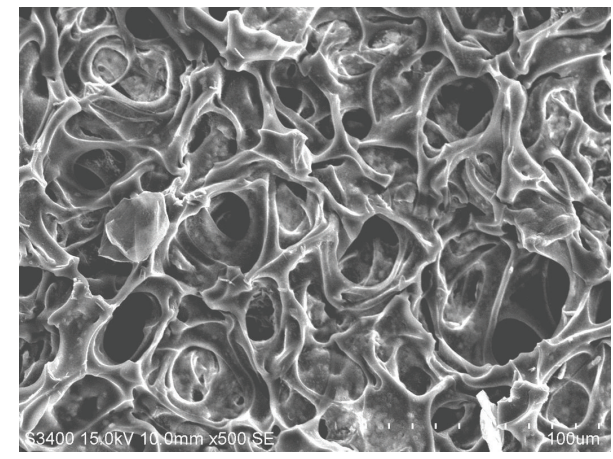

(a)

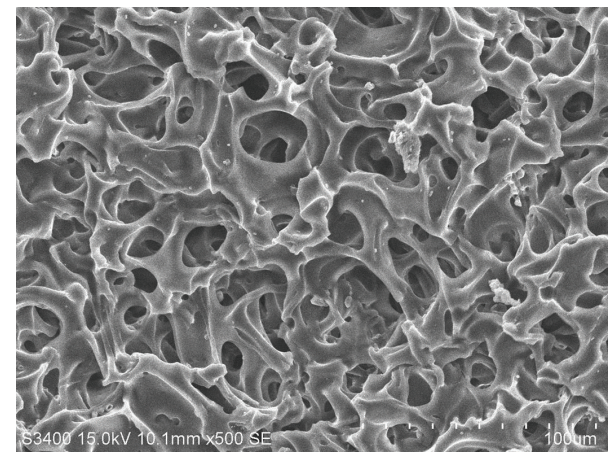

(b)

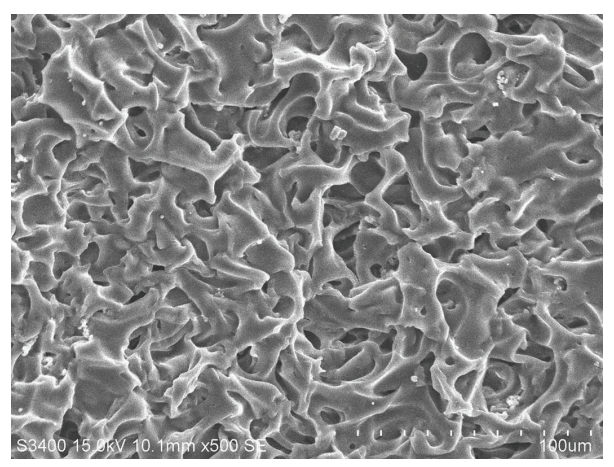

(c)

FIGURE 6: SEM image of degraded PVA-g-chitosan 3D scaffolds (CH50) by enzyme ((a) original material, (b) lipase, and (c) lysozyme).

3.5. Biodegradability of 3D Scaffold. For tissue engineering, the ultimate goal for applications of PVA-g-chitosan 3D scaffolds is natural disintegration as the cells grow. The time to degradation naturally affects cell growth conditions. The environment of the human body was simulated by using the enzymes lipase and lysozyme to evaluate the biodegradability of the scaffold. Both enzymes are hydrolases. Lipase is a lipid-digesting enzyme that mainly affects carbonyl groups, whereas lysozyme is a carbohydratedigesting enzyme that can hydrolyze the bindings between $\mathrm{N}$-acetylmuramic acid and $\mathrm{N}$-acetylglucosamine in some bacterial cell walls. Therefore, in this study, the enzymes lipase and lysozyme were used as degradation enzymes to investigate the time course of degradation of 3D scaffolds.

Figure 5 shows the enzymatic degradation experiments with scaffolds of various chitosan concentrations. The figure shows that the weight loss of PVA-g-chitosan 3D scaffold (CH25, CH50, and CH75) all increases as time increases. Moreover, degradation slightly decelerates as chitosan concentration increases. For example, the weight loss in the $\mathrm{CH} 75$ PVA-g-chitosan 3D scaffolds (-) was almost $10 \%$ after 35 days. A possible explanation for the enzyme degradation rate 


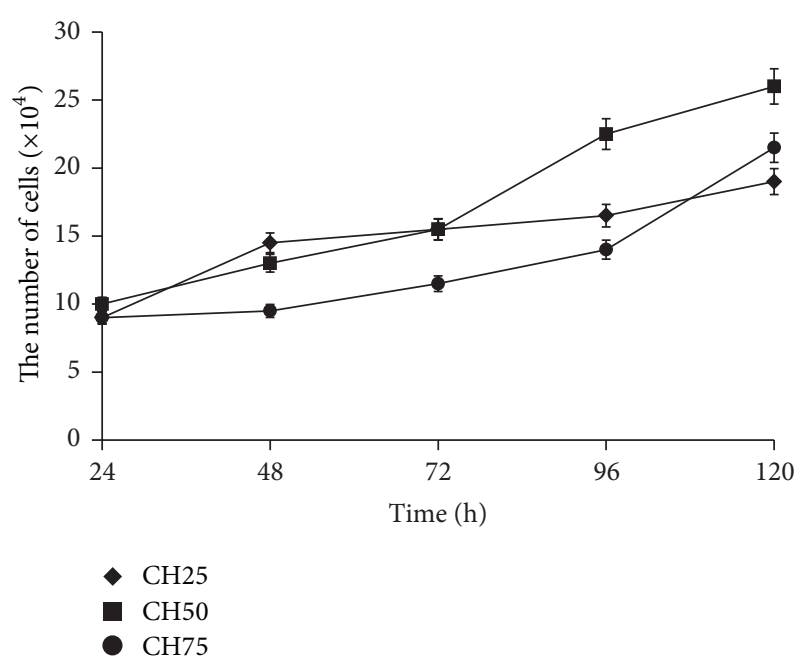

FIGURE 7: Cell proliferation of NIH3T3 fibroblasts cell on various grafted PVA-g-chitosan 3D scaffolds as a function of time.

of chitosan scaffolds is that only approximately $10 \%$ of the functional active sites are related to lysozyme and lipase. Most of its functional sites are $\beta$-(1-4)-glycosidic bonds between polysaccharides. The deacetylation of the chitosan used in this research approximated $75 \%$. Moreover, the grafting of PVA and chitosan also affected the degradation rate in the scaffolds, and the weight of chitosan scaffolds at equilibrium is reduced by $10 \%$. The PVA-g-chitosan $3 \mathrm{D}$ scaffolds (CH25) without enzyme added in the PBS solution $(\boldsymbol{\Delta})$ revealed a weight loss approximating 3\%, which was consistent with other reports [26].

Figure 6 shows a SEM micrograph of a chitosan scaffold (CH50) after 35 days of enzymatic degradation at $37^{\circ} \mathrm{C}$. Figures 6(b) and 6(c) clearly show the partial breakdown of the PVA-g-chitosan 3D scaffolds and surface degradation after enzymatic treatment for 35 days. These experimental results suggest that the grafted $3 \mathrm{D}$ scaffold can be degraded by enzymes in vivo and can thus be used in tissue engineering.

3.6. In Vitro Test. To elucidate the cell culturing conditions of a 3D scaffold, mouse NIH3T3 fibroblasts were implanted into a 3D scaffold, and the consequent cell growth was monitored. Figure 7 plots the growth curves of the mouse NIH3T3 fibroblasts cells implanted in the 3D scaffold. As the culture time increased, the cell number increased up to a maximum of 15 to $25 \times 10^{4}$ in 120 hours, which indicated that the material supported cell proliferation. Figure 8 shows electron microscope SEM and EDS diagrams of cells implanted in the $3 \mathrm{D}$ scaffold (b) in comparison with those in the original 3D scaffold (a). The photograph reveals significant cell growth. The EDS analysis revealed the presence of phosphorus and calcium, which confirmed the successful growth of cells on the grafted PVA-g-starch 3D scaffold.

\section{Conclusions}

This study developed a simple method of manufacturing PVA-g-chitosan 3D scaffolds. The main purpose of this study

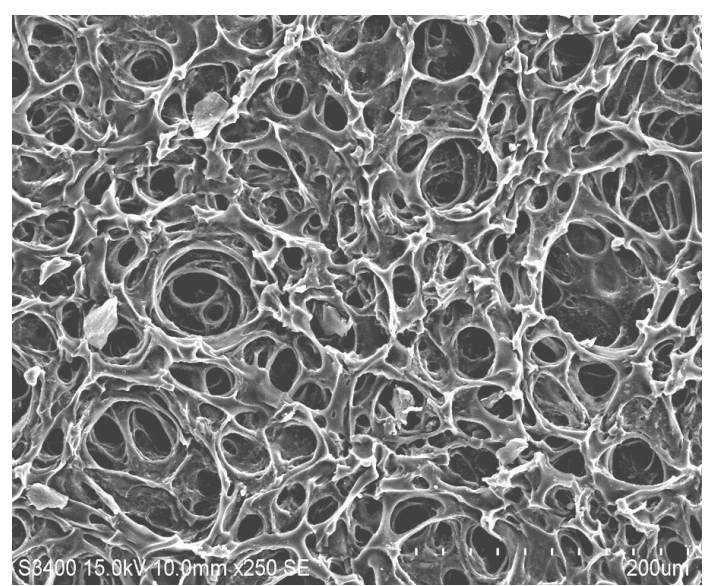

(a)
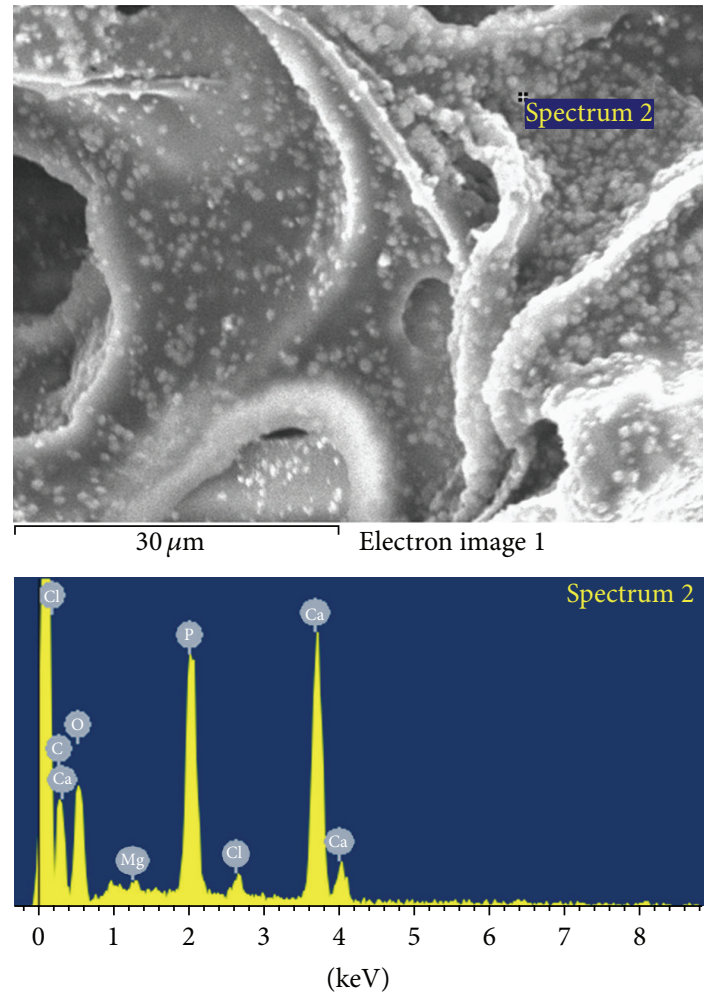

Full scale 420 cts cursor: 0.000

(b)

FIGURE 8: SEM images of NIH3T3 fibroblasts cells on grafted PVAg-chitosan 3D scaffolds after 5 days of culture ((a) original material, (b) materials with cells, $\times 3000$ and EDS analysis).

was to investigate the feasibility of using a PVA-g-chitosan 3D scaffold for cell culture in tissue engineering. After uniformly mixing the chitosan and PVA solution with a homogenizer at $300 \mathrm{rpm}, \mathrm{H}_{2} \mathrm{SO}_{4}$ and formaldehyde were added for catalysis. The PVA-g-chitosan was successfully prepared. The porosities of all three different chitosan concentrations used to form the $3 \mathrm{D}$ scaffold exceeded $72 \%$, but porosity decreased as the chitosan concentration increased. The water absorption of the 3D scaffold was dependent on the chitosan concentration, 
which demonstrated the influence of the chitosan concentration on the amount of water absorbed. The $3 \mathrm{D}$ scaffold has excellent mechanical strength. The compression strength of CH25 PVA-g-chitosan 3D scaffolds reached $16 \times 10^{-1} \mathrm{MPa}$. The degradation ratio of the 3D PVA-g-chitosan scaffold is low and is dependent on deacetylation and grafting. The PVA-g-chitosan 3D scaffold is highly compatible with cell attachment. As the culture time increased, the number of cells also increased up to a maximum of 15 to $25 \times 10^{4}$ in 120 hours.

\section{Conflict of Interests}

The authors declare that there is no conflict of interests regarding the publication of this paper.

\section{References}

[1] H. S. Mansur and H. S. Costa, "Nanostructured poly(vinyl alcohol)/bioactive glass and poly(vinyl alcohol)/chitosan/bioactive glass hybrid scaffolds for biomedical applications," Chemical Engineering Journal, vol. 137, no. 1, pp. 72-83, 2008.

[2] L. V. Thomas, U. Arun, S. Remya, and P. D. Nair, "A biodegradable and biocompatible PVA-citric acid polyester with potential applications as matrix for vascular tissue engineering," Journal of Materials Science: Materials in Medicine, vol. 20, no. 1, pp. S259-S269, 2009.

[3] S. H. Oh, S.-G. Kang, E.-S. Kim, S.-H. Cho, and J.-H. Lee, "Fabrication and characterization of hydrophilic poly(lactic-coglycolic acid)/poly(vinyl alcohol) blend cell scaffolds by meltmolding particulate-leaching method," Biomaterials, vol. 24, no. 22, pp. 4011-4021, 2003.

[4] F. Croisier and C. Jérôme, "Chitosan-based biomaterials for tissue engineering," European Polymer Journal, vol. 49, no. 4, pp. 780-792, 2013.

[5] H. Deng, X. Li, B. Ding et al., "Fabrication of polymer/layered silicate intercalated nanofibrous mats and their bacterial inhibition activity," Carbohydrate Polymers, vol. 83, no. 2, pp. 973-978, 2011.

[6] A. R. C. Duarte, J. F. Mano, and R. L. Reis, "Novel 3D scaffolds of chitosan-PLLA blends for tissue engineering applications: preparation and characterization," The Journal of Supercritical Fluids, vol. 54, no. 3, pp. 282-289, 2010.

[7] C. Ji, N. Annabi, A. Khademhosseini, and F. Dehghani, "Fabrication of porous chitosan scaffolds for soft tissue engineering using dense gas $\mathrm{CO}_{2}$," Acta Biomaterialia, vol. 7, no. 4, pp. 16531664, 2011.

[8] H. Liao, R. Qi, M. Shen et al., "Improved cellular response on multiwalled carbon nanotube-incorporated electrospun polyvinyl alcohol/chitosan nanofibrous scaffolds," Colloids and Surfaces B: Biointerfaces, vol. 84, no. 2, pp. 528-535, 2011.

[9] P. A. L. Lima, C. X. Resende, G. D. de Almeida Soares, K. Anselme, and L. E. Almeida, "Preparation, characterization and biological test of 3D-scaffolds based on chitosan, fibroin and hydroxyapatite for bone tissue engineering," Materials Science and Engineering C, vol. 33, no. 6, pp. 3389-3395, 2013.

[10] H. Deng, P. Lin, S. Xin et al., "Quaternized chitosan-layered silicate intercalated composites based nanofibrous mats and their antibacterial activity," Carbohydrate Polymers, vol. 89, no. 2, pp. 307-313, 2012.

[11] J. Ma and Y. Sahai, "Chitosan biopolymer for fuel cell applications," Carbohydrate Polymers, vol. 92, no. 2, pp. 955-975, 2013.
[12] N. Mohan and P. D. Nair, "Polyvinyl alcohol-poly(caprolactone) semi IPN scaffold with implication for cartilage tissue engineering," Journal of Biomedical Materials Research Part B: Applied Biomaterials, vol. 84, no. 2, pp. 584-594, 2008.

[13] N. Mohan, P. D. Nair, and Y. Tabata, "Growth factor-mediated effects on chondrogenic differentiation of mesenchymal stem cells in 3D semi-IPN poly(vinyl alcohol)-poly(caprolactone) scaffolds," Journal of Biomedical Materials Research, Part A, vol. 94, no. 1, pp. 146-159, 2010.

[14] S. A. Poursamar, M. Azami, and M. Mozafari, "Controllable synthesis and characterization of porous polyvinyl alcohol/hydroxyapatite nanocomposite scaffolds via an in situ colloidal technique," Colloids and Surfaces B: Biointerfaces, vol. 84, no. 2, pp. 310-316, 2011.

[15] S. Jiang, S. Liu, and W. Feng, "PVA hydrogel properties for biomedical application," Journal of the Mechanical Behavior of Biomedical Materials, vol. 4, no. 7, pp. 1228-1233, 2011.

[16] A. S. Asran, S. Henning, and G. H. Michler, "Polyvinyl alcoholcollagen-hydroxyapatite biocomposite nanofibrous scaffold: mimicking the key features of natural bone at the nanoscale level," Polymer, vol. 51, no. 4, pp. 868-876, 2010.

[17] L. Li and Y.-L. Hsieh, "Chitosan bicomponent nanofibers and nanoporous fibers," Carbohydrate Research, vol. 341, no. 3, pp. 374-381, 2006.

[18] L. Nie, D. Chen, J. Suo et al., "Physicochemical characterization and biocompatibility in vitro of biphasic calcium phosphate/polyvinyl alcohol scaffolds prepared by freeze-drying method for bone tissue engineering applications," Colloids and Surfaces B: Biointerfaces, vol. 100, pp. 169-176, 2012.

[19] Z. Osman and A. K. Arof, "FTIR studies of chitosan acetate based polymer electrolytes," Electrochimica Acta, vol. 48, no. 8, pp. 993-999, 2003.

[20] Y. Pranoto, S. K. Rakshit, and V. M. Salokhe, "Enhancing antimicrobial activity of chitosan films by incorporating garlic oil, potassium sorbate and nisin," LWT-Food Science and Technology, vol. 38, no. 8, pp. 859-865, 2005.

[21] C.-T. Lee, P.-H. Kung, and Y.-D. Lee, "Preparation of poly(vinyl alcohol)-chondroitin sulfate hydrogel as matrices in tissue engineering," Carbohydrate Polymers, vol. 61, no. 3, pp. 348-354, 2005.

[22] C. Xiao and M. Yang, "Controlled preparation of physical crosslinked starch-g-PVA hydrogel," Carbohydrate Polymers, vol. 64, no. 1, pp. 37-40, 2006.

[23] W.-C. Hsieh and J.-J. Liau, "Cell culture and characterization of cross-linked poly(vinyl alcohol)-g-starch 3D scaffold for tissue engineering," Carbohydrate Polymers, vol. 98, no. 1, pp. 574-580, 2013.

[24] N. Bhardwaj and S. C. Kundu, "Silk fibroin protein and chitosan polyelectrolyte complex porous scaffolds for tissue engineering applications," Carbohydrate Polymers, vol. 85, no. 2, pp. 325-333, 2011.

[25] W.-C. Hsieh, C.-P. Chang, and S.-M. Lin, "Morphology and characterization of 3D micro-porous structured chitosan scaffolds for tissue engineering," Colloids and Surfaces B: Biointerfaces, vol. 57, no. 2, pp. 250-255, 2007.

[26] S. N. Alhosseini, F. Moztarzadeh, M. Mozafari et al., "Synthesis and characterization of electrospun polyvinyl alcohol nanofibrous scaffolds modified by blending with chitosan for neural tissue engineering," International Journal of Nanomedicine, vol. 7, pp. 25-34, 2012. 

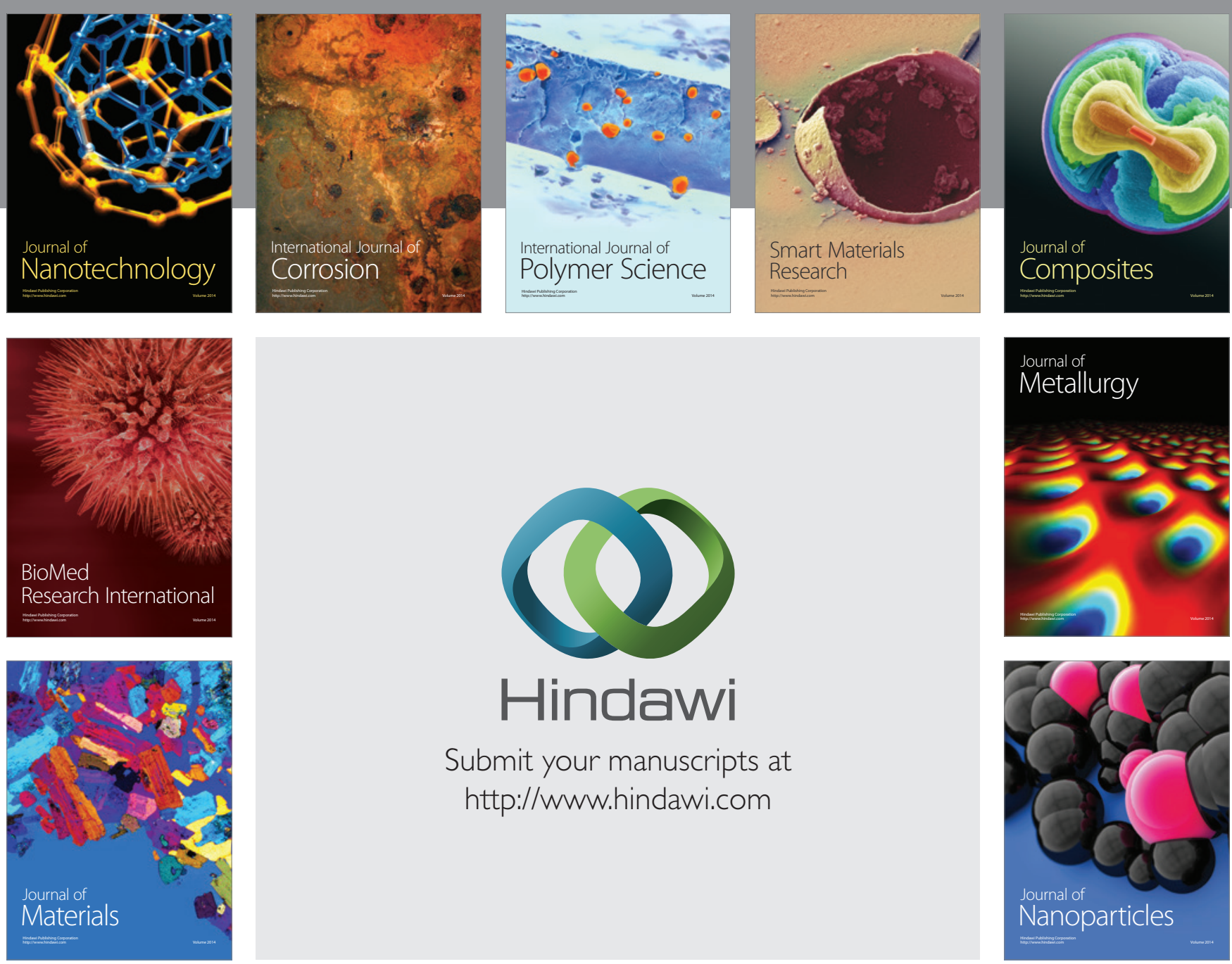

Submit your manuscripts at http://www.hindawi.com
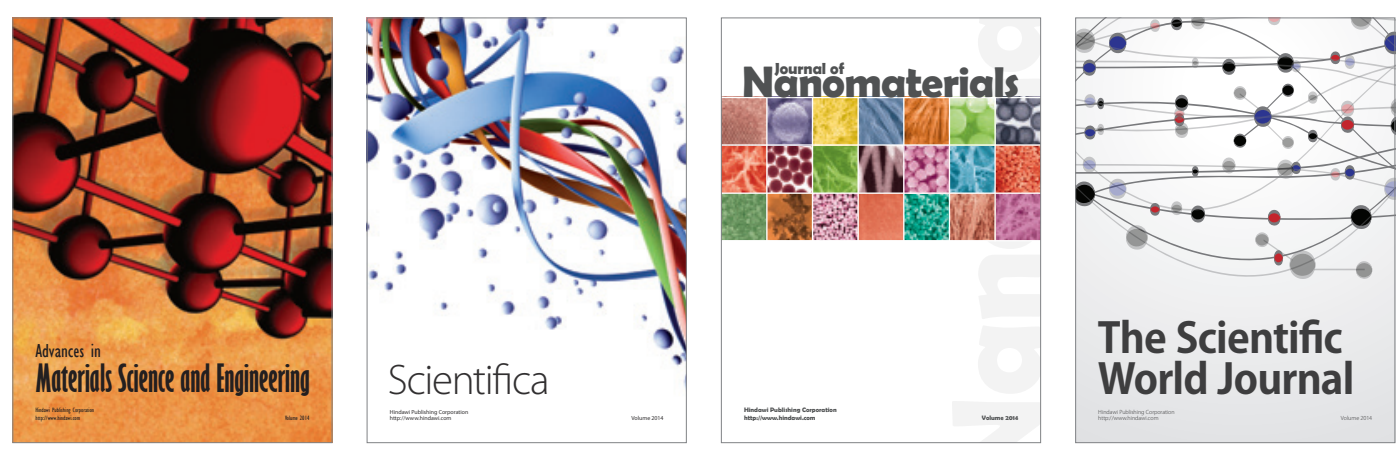

\section{The Scientific World Journal}
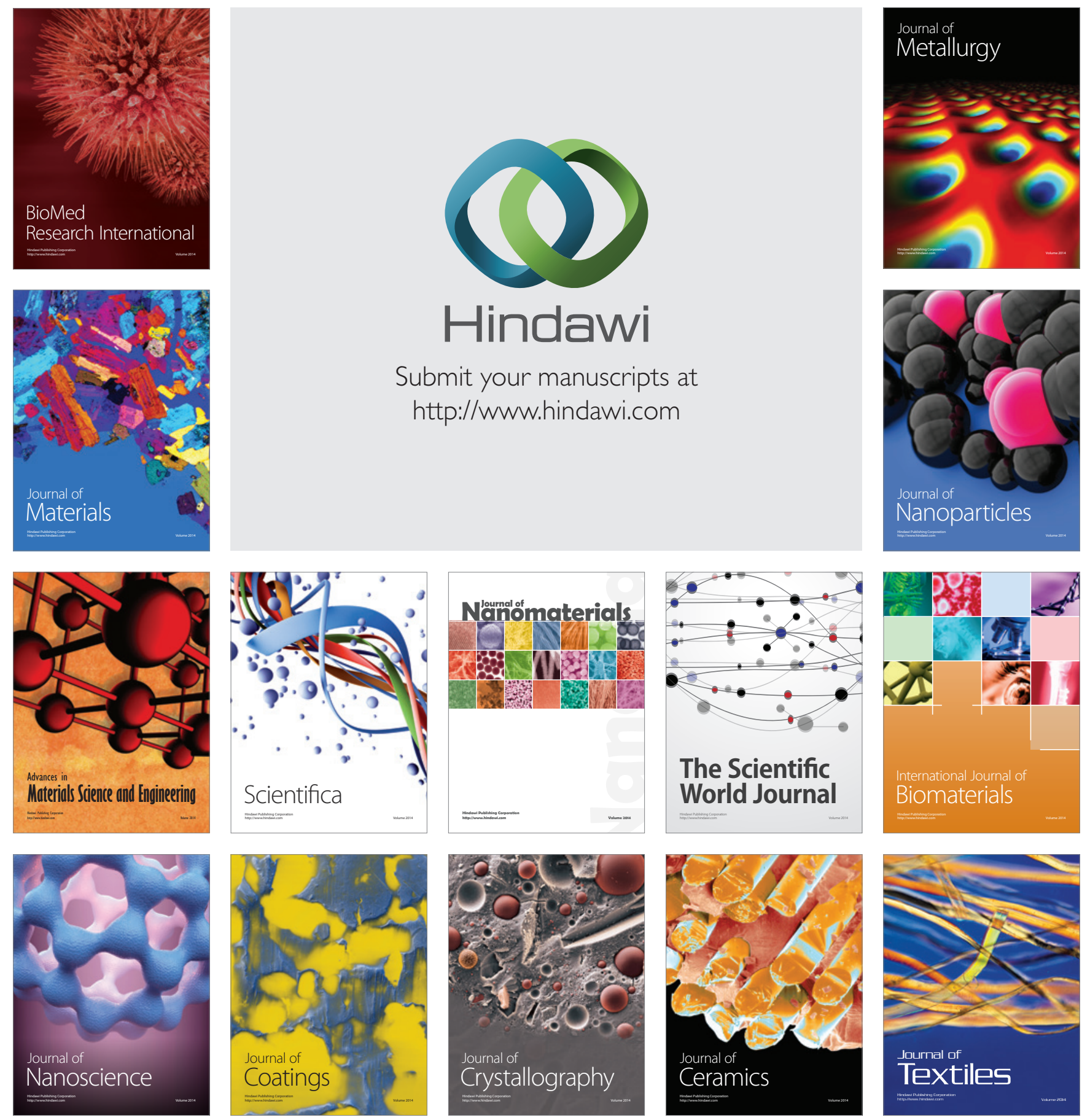